Publ. RIMS, Kyoto Univ. Ser. A

Vol. 4 (1969), pp. 595-609

\title{
On Projective Invariance of Brownian Motion
}

\author{
By \\ Takeyuki Hida,* Izumi Kubo, * Hisao Nomoto* \\ and Hisaaki Yoshizawa ${ }^{\dagger}$
}

\begin{abstract}
Let $\boldsymbol{E}$ be a certain nuclear topological vector space contained in the Hilbert space $\left(L^{2}\right)$ on the real line and let the Gaussian probability measure be defined on the conjugate space $E^{*}$ of $E$. We consider such a subgroup of the rotation group of $\left(L^{2}\right)$ that acts on $E$ and contains the shift as a one-parameter subgroup. With a rather systematic way we define two more one-parameter subgroups which, together with the shift, constitute a subgroup isomorphic with the projective linear group $P G L(2, \boldsymbol{R})$. It plays a role of the time change of the white noise. In this set up we formulate and prove the principle of projective invariance of the Brownian motion given by P. Lévy.
\end{abstract}

\section{§1. Introduction}

The purpose of this paper is to investigate a specific class of oneparameter groups of orthogonal operators acting on $L^{2}(\boldsymbol{R})$, the Hilbert space of real-valued square integrable functions on the real line $\boldsymbol{R}$, and its relation with some probabilistic properties of the Brownian motion. For brevity we shall denote the Hilbert space $L^{2}(\boldsymbol{R})$ by $\mathfrak{W}$ throughout the paper.

For our purpose, we first consider some subgroups of the group of all orthogonal operators acting on $\mathfrak{W}$, as follows:

Let $E$ be a topological vector space contained in $\mathfrak{D}$ and its topology be stronger than the norm topology of $\mathfrak{W}$. Then we have the relations

Received November 21, 1968.

Communicated by S. Matsuura.

*Department of Mathematics, Nagoya University.

† Department of Mathematics, Kyoto University. 


$$
E \subset \mathfrak{g} \subset E^{*}
$$

where $E^{*}$ is the conjugate space of $E$. An orthogonal openator $g$ of $\mathfrak{S}$ is called a rotation of $E$, if $g$ mars $E$ onto itself and is a homeomorphism of $E$. The collection of all such $g$ 's forms a group which we denote by $O(E ; \mathfrak{Q})$ or simply by $O(E)$ and call the rotation group of $E$.

The second fundamental object which we co-sider in this paper is the so-called white noise; it is a probability measure $\mu$ on $E^{*}$ and its characteristic functional (or, what is the same, its Fourier transform) is equal to

$$
C(\xi)=\mathrm{e}^{-\frac{1}{2}\|\xi\|^{2}}, \quad \text { for } \xi \text { of } E,
$$

where $\|\cdot\|$ stands for the norm of $\mathfrak{g}$. The characteristic functional of $\mu$ is defined as usual:

$$
C(\xi)=\int_{E^{*}} \mathrm{e}^{i x, \xi} d \mu(x),
$$

where $\langle x, \xi\rangle$ stands for the canonical bilinear form on $E^{*} \times E$.

The functional $C(\cdot)$, defined by the equality $(\mathrm{A})$, is continuous and positive definite in $E$, therefore, there exists a probability measure $\mu$ on $E^{*}$ satisfying the relation (B), in case the vector space $E$ is nuclear (see, for example, [1]), which we assume throughout the paper. As is easily seen from the expression of the characteristic functional $C(\cdot)$, it is invariant under the action of $g$ of $O(E)$. Hence the measure $\mu$ is invariant under $g^{*}$, the adjoint operator of $g$, acting on the space $E^{*}$. Moreover, it is known that $\mu$ is ergodic with respect to the group $O^{*}(E)=\left\{g^{*} ; g \in O(E)\right\}$.

Now we consider dynamical systems in the above set up. Given a one-parameter subgroup $\left(g_{t}\right)$ of $O(E)$, we can define a flow, or a dynamical system $\left(g_{t}^{*}\right),-\infty<t<\infty$, on the measure space $\left(E^{*}, \mu\right)$. The simplest and most basic of such is the shift:

$$
\sigma_{t}: \xi(u) \mid \rightarrow \xi(u-t), \quad-\infty<t<\infty .
$$

The corresponding flow on $\left(E^{*}, \mu\right)$ is nothing but the flow of the Brownian motion. This situation will be clarified by Proposition 4 in 
Section 4. Generalizing the concept of the shift we are led to consider an important class of one-parameter groups which come from the change of the variable of functions $\xi(u)$ having the form:

$$
\xi(u) \mid \rightarrow \xi\left(\psi_{t}(u)\right) \sqrt{\left|\psi_{t}^{\prime}(u)\right|},
$$

with the relation $\psi_{t} \circ \psi_{s}(u)=\psi_{t+s}(u)$.

All these considerations, which are preliminary to our principal purpose, are summarized in Section 2.

In Section 3 we shall show that, starting with the shift, we obtain an interesting class of one-parameter subgroups which form a three dimensional subgroup $G_{0}$ of $O(E)$. Here the nuclear space $E$ is taken in accordance with the group $G_{0}$, and the group $G_{0}$ is isomorphic with the group $P G L(2, \boldsymbol{R})$, the group of all projective linear transformations in real two dimension. In the course of our study to determine the group $G_{0}$ we appeal to the usual technique of Lie algebra.

In Section 4 we shall apply our constructions to a theory of the Brownian motion. Namely, we show that in our set up, the fact that the group $O(E)$ admits the subgroup $G_{0}$ gives us a rephrase of the principle of projective invariance of the Brownian motion discovered by P. Lévy.

This principle is illustrated as follows: Given a Brownian motion $B(t), 0 \leqq t<\infty$, we can speak of a pinned Brownian motion, say pinned at the moments $t_{0}$ and $t_{1}\left(0 \leqq t_{0} \leqq t_{1}\right)$ in such a way that $B\left(t_{0}\right)=B\left(t_{1}\right)$ $=0$. Denote such a process by $X(t), t_{0} \leqq t \leqq t_{1}$, and normalize it so as to obtain a Gaussian process $Y(t), t_{0} \leqq t \leqq t_{1}$; that is, put

$$
Y(t)=\frac{X(t)}{\sqrt{V(X(t))}},
$$

where $V(\cdot)$ denotes the variance. Now let $g$ be a projective tranrformation of the interval $\left[t_{0}, t_{1}\right]$ onto an interval. Then the process $Y_{g}(t)$ $=Y(g t), t_{0} \leqq t \leqq t_{1}$, is the same process as the original process $Y(t)$. The process $Y(t)$ can be realized as a system of random variables on the probability space $\left(E^{*}, \mu\right)$ and the action by the above $g$ can be represented by a member of the group $G_{0}$. 
The group $O(E)$ was considered, among similar objects, by the last named author of the present paper in 1961, and some results on its structure and unitary representations have been given in series of lectures. They will be published on other occasions. The authors of the present paper had a series of seminars in 1967 at the Research Institute for Mathematical Sciences, Kyoto University, and the results in the present paper, together with other several related topics, were discussed. The first named author has investigated the subjects of Section 4 and other similar problems. They will be published in another paper.

\section{§2. Rotation Group of Hilbert Space}

In this section we shall give fundamental definitions and some preliminary results used throughout the paper. In particular we shall define a dynamical system in general, the rotation group of the Hilbert space and the white noise.

Let $(M, m)$ be a measure space with a $\sigma$-finite measure $m$. A mapping $\varphi$ from $M$ to $M$ is said to be nonsingular (or absolutely continuous), if

(i) both $\varphi$ and $\varphi^{-1}$ are defined on $M$ modulo $m$-null sets;

(ii) they are measurable;

(iii) they carry every $m$-null set into an $m$-null set.

A dynamical system $\left(\varphi_{t}\right),-\infty<t<\infty$, on $(M, m)$ is a one-parameter family of nonsingular transformations $\varphi_{t}$ such that

$$
\varphi_{s} \varphi_{t}=\varphi_{s+t} \quad(\bmod 0) \text { for each } t \text { and } s,
$$

and that $\varphi_{t} u$ is measurable in $(t, u)$.

Let $L^{2}(M)$ be the Hilbert space of all square $m$-integrable real functions on $M$ with the inner product

$$
(f, h)=\int_{M} f h d m
$$

We denote by $O\left(L^{2}(M)\right)$ the group of all orthogonal operators acting on $L^{2}(M)$. A dynamical system $\left(\varphi_{t}\right)$ on $M$ generates a one-parameter subgroup $\left(F_{t}\right)$ of $O\left(L^{2}(M)\right)$ in the following manner: 


$$
F_{t}: f(u) \mid \rightarrow f\left(\varphi_{t} u\right) \sqrt{\frac{d m\left(\varphi_{t} u\right)}{d m(u)}} \text {, for } f \text { of } L^{2}(M) \text {. }
$$

It satisfies the group property:

$$
F_{s} F_{t}=F_{s+t} \text {. }
$$

The mapping $t \rightarrow F_{t} f$ is strongly continuous for every $f$ because $(t, u) \mapsto \varphi_{t} u$ is measurable. Thus we can define the generator $A^{\varphi}$ of the one-parameter group $\left(F_{t}\right)$ by the following formula:

$$
A^{\Phi} f=\lim _{t \rightarrow 0} \frac{F_{t} f-f}{t} \text {, if the strong limit exists. }
$$

If both $f$ and $h$ belong to the domain of $A^{s}$, then the relation $\left(F_{t} f, h\right)=\left(f, F_{-t} h\right)$ implies that

$$
\left(A^{\rho} f, h\right)+\left(f, A^{\rho} h\right)=0 .
$$

Suppose we are given a dense linear subspace $E$ of $L^{2}(M)$ such that $E$ is a nuclear topological vector space and the inner product $(f, h)$ is continuous on $E$. Now let $G$ be a subgroup of $O\left(L^{2}(M)\right)$. We say that $E$ is stable under $G$ if each $g$ of $G$ leaves $E$ invariant. In this case $g$ is a homeomorphism of $E$. We denote by $O(E)$ the set of all such $g$ 's; it is a subgroup of $O\left(L^{2}(M)\right)$. We do not consider topology of $O(E)$ in the present paper.

Consider the functional

$$
C(\xi)=\mathrm{e}^{-\frac{1}{2}|| \xi \|^{2}}, \quad \xi \text { in } E .
$$

Then by Bochner-Minlos theorem, there exists such a probability measure $\mu$ on the conjugate space $E^{*}$ of $E$ that $C(\xi)$ is the characteristic functional of $\mu$ :

$$
C(\xi)=\int_{E^{*}} \mathrm{e}^{i \wedge \wedge, \xi} d \mu(x) .
$$

The measure $\mu$ is called the white noise.

Now we have the following proposition which is proved easily.

Proposition 1. Let $\mu$ be the white noise on $E^{*}$. Then

(i) If $\left(g_{t}\right)$ is a continuous one-parameter subgroup of $O(E)$, 
then $\left(g_{t}^{*}\right)$ is a flow on $\left(E^{*}, u\right)$, where $g_{t}^{*}$ denotes the adjoint operator of $g_{t}$ :

$$
\left\langle g_{t}^{*} x, \xi\right\rangle=\left\langle x, g_{t} \xi\right\rangle \text {, for } x \text { of } E^{*} \text { and } \xi \text { of } E \text {. }
$$

(ii) If we define the mapping $r$ from $E$ into $L^{2}\left(E^{*}, \mu\right)$ by

$$
\gamma: \xi \mid \rightarrow \gamma(\xi)=\langle x, \xi\rangle,
$$

then $r$ can be extended to a mapping which defines a linear isometry from $L^{2}(M)$ into $L^{2}\left(E^{*}, \mu\right)$. Moreover, each image $r(f)$ of $f$ in $L^{2}(M)$ (we shall denote it also by $\langle x, f\rangle$ ) is a Gaussian random variable with mean 0 and variance $\|f\|^{2}$.

\section{§3. Projective Linear Group}

In what follows, we shall consider the case where $M$ is the real line $\boldsymbol{R}$ and $m$ is the Lebesgue measure. We shall denote $m(d u)$ simply by $d u$ and $L^{2}(\boldsymbol{R})$ by $\mathfrak{S}$. In this section we shall define a specialized nuclear space $E$ and consider a subgroup of $O(E)$, isomorphic with the real projective linear group $P G L(2, \mathbb{R})$. They are utilized in the following section.

To begin with, we consider the shift $\left(\sigma_{t}\right)$ which is a dynamical system on $\mathbb{R}$ defined by

$$
\sigma_{t}: u \mid \rightarrow u-t
$$

The corresponding one-parameter group $\left(S_{t}\right)$ on $\mathfrak{g}$ and its generator $A^{\sigma}$ are expressed in the following forms:

$$
S_{t}: f(u) \mid \rightarrow f\left(\sigma_{t} u\right)=f(u-t)
$$

and

$$
A^{\sigma}: f(u) \mid \rightarrow-\frac{d}{d u} f(u) \text { for smooth functions } f
$$

Our aim is to find dynamical systems on $\mathbb{R}$ which are defined by the change of variable $u$ and generalize the concept of the shift. We can give the following two dynamical systems as examples. The first one is given by

$$
\tau_{t}: u \mid \rightarrow u \mathrm{e}^{t}
$$


which we shall call the tension. The corresponding operators have the form

$$
T_{t}: f(u) \mid \rightarrow f\left(u \mathrm{e}^{t}\right) \mathrm{e}^{t / 2}
$$

The second one is given by

$$
\kappa_{t}: u \mid \rightarrow \frac{u}{1-u t}
$$

and the corresponding operators have the form

$$
K_{t}: f(u) \mid \rightarrow f\left(\frac{u}{1-u t}\right) \frac{1}{|1-u t|} .
$$

We are interested in finding, in a systematic way, a more general class of dynamical systems which fulfill commutation relations each other. For this purpose we consider the collection a of all $C^{\infty}$-functions $a(u)$ on $\boldsymbol{R}$, which becomes a Lie algebra with the product:

$$
[a, b]=a \frac{d}{d u} b-b \frac{d}{d u} a .
$$

For each function $a(\cdot)$ in a, we define the differential operator

$$
D(a) \cdot=a(u) \frac{d}{d u} \cdot+\frac{1}{2} a^{\prime}(u) \cdot
$$

Then, under some regularity conditions, the generator of a dynamical system $\left(\varphi_{t}\right)$ is expressed in the form

$$
A^{\Phi}=D(a), \text { where } a(u)=\left.\frac{d\left(\varphi_{t} u\right)}{d t}\right|_{t=0} .
$$

In case where the system $\left(\varphi_{t}\right)$ is the shift $\left(\sigma_{t}\right)$ or the tension $\left(\tau_{t}\right)$,

$$
a(u)=-1 \text { or } a(u)=u \text {, respectively. }
$$

Here arises a problem: For which function $a(\cdot)$ the operator $D(a)$ could be a generator of a dynamical system? Concerning this question, we can prove the following proposition:

Proposition 2. Denote by $a_{n}(n=1,2, \cdots)$ any possible n-dimensional subalgebra of the Lie algebra a spanned by 1 and $n-1$ polynomials. Then there are only two possible cases; more precisely, 
(i) The monomials 1 and $u$ form a base of a two-dimensional $\mathfrak{a}_{2}$ with the relation

$$
[-1, u]=-1
$$

the operator $D(u)$ (defined by formula (14)) corresponds to the generator of the tension $\left(\tau_{t}\right)$.

(ii) The monomials $1, u$ and $u^{2}$ form a base of a three-dimensional $a_{3}$ with the relations

$$
\left[1, u^{2}\right]=2 u, \quad\left[u, u^{2}\right]=u^{2},
$$

and we have

$$
D\left(u^{2}\right)=A^{\kappa}
$$

(iii) For $n>3$, there exists no subalgebra $a_{n}$.

Proof. Let functions 1 and $a(\cdot)$ form a base of a subalgebra $a_{2}$. Then $[1, a]=a^{\prime}$ and it has to be a linear combination of 1 and $a$, that is, $a^{\prime}=\alpha+\beta a$. The solution of this differential equation is

$$
a(u)=\alpha u \quad \text { or } \quad a(u)=\gamma \mathrm{e}^{\beta u}-\frac{\alpha}{\beta},
$$

according as $\beta=0$ or $\neq 0$. In the latter case the function $a(\cdot)$ is not a polynomial, therefore we should choose the functions 1 and $u$ as a base of $a_{2}$.

The case of an algebra $\mathfrak{a}_{3}$ can be treated in an analogous manner, and the last assertion can be proved easily.

Q.E. D.

Remark. Dynamical systems $\left(\sigma_{t}\right),\left(\tau_{t}\right)$ and $\left(\kappa_{t}\right)$ are related with each other in the following way:

$$
\begin{aligned}
\tau_{t} \sigma_{s} & =\sigma_{s \exp t} \tau_{t}, \\
\tau_{t} \kappa_{s} & =\kappa_{s \exp (-t)} \tau_{t}, \\
\kappa_{t} & =\lambda^{-1} \sigma_{t} \lambda,
\end{aligned}
$$

where $\lambda$ is the non-singular transformation defined by

$$
\lambda: u \mapsto \frac{1}{u}
$$


The operator $L$ associated with $\lambda$ by the formula (1) is given by

$$
L: f(u) \mapsto f\left(\frac{1}{\mathrm{u}}\right) \frac{1}{u} .
$$

The next step of our discussion is to find a function space $E$, which is contained in $\mathfrak{W}$ and is stable under the operators $\left(S_{t}\right),\left(T_{t}\right)$ and $\left(K_{t}\right)$; namely, we are looking for such a space $E$ that $\left(S_{t}\right),\left(T_{t}\right)$ and $\left(K_{t}\right)$ belong to the group $O(E)$. The following function space $D_{0}$, introduced by Gelfand and others [2], is fitting for our purpose:

$$
D_{0}=\left\{f(u) ; f(u) \in C^{\infty} \text { and } L f(u) \in C^{\infty}\right\},
$$

where $L$ is the operator defined in (21). The topology of $D_{0}$ is defined in the natural way. Then we have

Proposition 3. We can define a countable system of norms in the space $D_{0}$ so that $D_{0}$ becomes a countably Hilbertian nuclear space and the following relations hold:

$$
D_{0} \subset \mathfrak{g} \subset D_{0}^{*}
$$

Proof. We use an auxiliary function space $W$ which is the collection of all $C^{\infty}$-functions $f(\theta)$ on the circle $S^{1}$ with the diameter equal to 1 such that $f(\theta+\pi)=f(\theta)$. We introduce countably many Hilbertian norms $\|\cdot\|_{p}(p=0,1,2, \cdots)$ to $W$ as follows:

$$
\|f\|_{i p}^{2}=\sum_{j=0}^{p} \int_{-\pi / 2}^{\pi / 2}\left|\frac{d^{j}}{d \theta^{j}} f(\theta)\right|^{2} d \theta \text {, for } f \text { of } W .
$$

Let $W_{p}$ be the Hilbert space obtained by the completion of $W$ with respect to the $p$-th norm $\|\cdot\|_{p}$. Then the following functions in $W$ form a complete orthonormal system in $W_{p}$ :

$$
\begin{cases}\varphi_{p, k}(\theta)=\sqrt{2}\left(\pi \sum_{j=0}^{p}(2 k)^{2 j}\right)^{-1 / 2} \sin 2 k \theta & (k=1,2, \cdots), \\ \psi_{p, k}(\theta)=\sqrt{2}\left(\pi \sum_{j=0}^{p}(2 k)^{2 j}\right)^{-1 / 2} \cos 2 k \theta \quad(k=1,2, \cdots), \\ \psi_{p, 0}(\theta)=\frac{1}{\sqrt{\pi}} .\end{cases}
$$

By using this base, we can prove that the injection $W_{p+2} \rightarrow W_{p}$ is is 
nuclear transformation and that

$$
W=\bigcap_{p=0}^{\infty} W_{p} .
$$

Hence $W$ is nuclear.

Consider the linear transformation $\gamma$ from $W$ to $D_{0}$ given by

$$
\gamma: f(\theta) \mid \rightarrow(r f)(u)=\frac{1}{\sqrt{1+u^{2}}} f(\arctan u)
$$

Obviously $\gamma$ is a one-to-one and onto mapping, so that we can transfer the nuclear structure of the space $W$ to the space $D_{0}$. Thus we have proved that $D_{0}$ is a $\sigma$-Hilbert nuclear space. The relations $D_{0} \subset \mathfrak{W} \subset D_{0}^{*}$ are obvious.

Q.E.D.

Remark. The Hilbertian norms appeared in the proof of Proposition 3 are transformed by the mapping $\gamma$ into the following forms:

$$
\begin{aligned}
\|\xi\|_{p}^{2}=\sum_{j=0}^{p} \int_{-\infty}^{\infty}\left|\left(D\left(1+u^{2}\right)\right)^{j} \xi(u)\right|^{2} d u, \\
\quad \text { for } \xi \text { of } D_{0}, p=0,1, \cdots .
\end{aligned}
$$

Moreover, the system of elements of $D_{0}$ given by

$$
\left\{\begin{array}{r}
\xi_{p, k}(u)=\sqrt{2}\left(\pi \sum_{j=0}^{p}(2 k)^{2 j}\right)^{-1 / 2}\left(1+u^{2}\right)^{-k-\frac{1}{2}} \sum_{l=0}^{k-1}\left(\begin{array}{l}
2 k \\
2 l+1
\end{array}\right)(-1)^{l} u^{2 l+1} \\
(k=1,2, \cdots), \\
\eta_{p, k}(u)=\sqrt{2}\left(\pi \sum_{j=0}^{p}(2 k)^{2 j}\right)^{-1 / 2}\left(1+u^{2}\right)^{-k-\frac{1}{2}} \sum_{l=0}^{k}\left(\begin{array}{l}
2 k \\
2 l
\end{array}\right)(-1)^{l} u^{2 l} \\
(k=1,2, \cdots), \\
\eta_{p, 0}(u)=\frac{1}{\sqrt{\pi}}\left(1+u^{2}\right)^{-1 / 2}
\end{array}\right.
$$

is a complete orthonormal system of $D_{0, p}$ which is the Hilbert space obtained by the completion of $D_{0}$ with respect to the norm $\|\cdot\|_{p}$ given by (26). (See expression (24).)

Theorem 1 . Let $G_{0}$ be the subgroup of $O(\mathfrak{S})$ generated by oneparameter groups $\left(S_{2}\right),\left(T_{t}\right),\left(K_{t}\right)$ and the operator $L$ defined in (21). Then $D_{0}$ is $G_{0}$-stable; in other words, $G_{0}$ is a subgroup of $O\left(D_{0}\right)$. 
The proof is straightforward by tiac definitions of the subgroup $G_{0}$ and the space $D_{0}$.

We now come to obtaining an explicit expression of the subgroup $G_{0}$ in Theorem 1. Let

$$
g=\left[\begin{array}{ll}
\alpha & \beta \\
\gamma & \delta
\end{array}\right]
$$

be an element of $G L(2, \boldsymbol{R})$. With $g$ we associate a transformation $\bar{g}$ from $D_{0}$ to $\mathfrak{g}$ in the following manner:

$$
\bar{g}: \xi(u) \mid \rightarrow \xi\left(\frac{\alpha u+\beta}{\gamma u+\delta}\right) \frac{\sqrt{|\operatorname{det} g|}}{|\gamma u+\delta|} .
$$

Then we have

Theorem 2. The transformation $\bar{g}$ given by the formula (28) belongs to $O\left(D_{0}\right)$ for every matrix $g$ of $G L(2, \boldsymbol{R})$, and the mapping $g \mapsto \bar{g}$ defines an isomorphism of $P G L(2, \mathbb{R})$ and $G_{0}$.

Proof. Linear fractional transformations $\sigma_{t}, \tau_{t}$ and $\kappa_{t}$ are defined by matrices

$$
\zeta(-t)=\left[\begin{array}{rr}
1 & -t \\
0 & 1
\end{array}\right], \quad \delta(t)=\left[\begin{array}{ll}
e^{t / 2} & 0 \\
0 & e^{-t / 2}
\end{array}\right] \quad \text { and } \quad z(-t)=\left[\begin{array}{rr}
1 & 0 \\
-t & 1
\end{array}\right]
$$

of $G L(2, \boldsymbol{R})$, respectively; and the transformation $\lambda$ given in the formula (20) corresponds to the matrix

$$
s=\left[\begin{array}{ll}
0 & 1 \\
1 & 0
\end{array}\right]
$$

On the other hand every matrix $g$ of $G L(2, \boldsymbol{R})$ is expressed, modulo the center, in either of the following way:

$$
g=\zeta(t) \delta(u) z(v) \text { or } g=s \zeta(t) \delta(u) z(v) .
$$

Therefore the mapping $g \mid \rightarrow \bar{g}$ is a homomorphism from $G L(2, \boldsymbol{R})$ onto $G_{0}$. The kernel of this mapping coincides with the center of $G L(2, \boldsymbol{R})$, from which follows the assertion of the theorem. 


\section{\$4. Projective Invariance}

This section will be devcted to an investigation of the projective invariance of the Brownian motion due to P. Léry [3] by using our discussion in Section 3.

We start with the nuclear space $D_{0}$ and the white noise $\mu$ on $D_{0}^{*}$, the conjugate space of $D_{0}$.

Lemma. Let $g$ be an element of $O\left(D_{0}\right)$ and $f$ belong to $\mathfrak{S}_{2}$. Then the mapping $f \mapsto g f$ can be defined in such a way that

$$
\langle x, g f\rangle=\left\langle g^{*} x, f\right\rangle
$$

in $L^{2}\left(D_{0}^{*}, \mu\right)$ as functions of $x$.

Proof. The equation (29) is true for every $x$ if $f$ is in $D_{0}$. For $f$ in $\mathfrak{S}$ we take a sequence $\left(\xi_{n}\right)$ in $D_{0}$ which converges to $f$ in $\mathfrak{S}$. Then the sequence of functions in $x,\left(\left\langle x, g \xi_{n}\right\rangle\right)$, is convergent in $L^{2}\left(D_{0}^{*}, \mu\right)$. Denote the limit by the symbol $\langle x, g f\rangle$. On the other hand we have

$$
\left\langle g^{*} x, \xi_{n}\right\rangle=\left\langle x, g \xi_{n}\right\rangle,
$$

and the left-hand side has the limit $\left\langle g^{*} x, f\right\rangle$ in $L^{2}\left(D_{0}^{*}, \mu\right)$. Thus we have the equality (29).

Q.E. D.

Using our set up we have a realization of the Brownian motion.

Proposition 4. (i) The process defined by

$$
\begin{aligned}
B(t, x)= & \left\langle x, \chi_{(-\infty, 0]}\left(\sigma_{t} \cdot\right)-\chi_{(-\infty, 0]}(\cdot)\right\rangle,{ }^{1)} \\
& -\infty<t<\infty, \quad x \in D_{0}^{*},
\end{aligned}
$$

is a Brownian motion.

(ii) The process defined by

$$
U(t, x)=\left\langle T_{t}^{*} x, \chi_{[0,1]}\right\rangle, \quad-\infty<t<\infty,
$$

is an Ornstein-Uhlenbeck Brownian motion.

Proof. Both $(B(t))$ and $(U(t))$ are Gaussian systems with mean zero and their covariance functions are given by

1) The function $\chi_{A}$ denotes the indicator function of a subset $A$ of $\boldsymbol{R}$. 


$$
\int_{D_{0}^{*}} B(t, x) B(s, x) d \mu(x)=\frac{|t|+|s|-|t-s|}{2}
$$

and

$$
\begin{aligned}
\int_{D_{0} *} U(t, x) U(s, x) d \mu(x) & =\left(T_{t} \chi_{[0,1]}, T_{s} \chi_{[0,1]}\right) \\
& =\mathrm{e}^{-\frac{1}{2}|t-s|},
\end{aligned}
$$

respectively.

Q. E. D.

Remark. The relation (17) implies that

$$
\left(S_{s \exp t}\right) * T_{t}^{*}=T_{t}^{*} S_{s}^{*} .
$$

Hence we may say that the Brownian motion is a transversal of the Ornstein-Uhlenbeck Brownian motion.

We consider a continuous curve $\left(f_{t}\right)$ in the Hilbert space $\mathfrak{S}$ where $t$ runs on an interval $I$. The adjcint $g^{*}$ of an element $g$ of $O\left(D_{0}\right)$ is a metrical automorphism of $\left(D_{0}^{*}, \mu\right)$, therefore Lemma implies that the stochastic processes defined by

$$
\left\langle x, f_{t}\right\rangle ; \quad t \in I
$$

and

$$
\left\langle g^{*} x, f_{t}\right\rangle=\left\langle x, g f_{i}\right\rangle ; \quad t \in I
$$

have the same probability law. Now let $\mathscr{F}$ be a family of curves

$$
f(u ; t ; a, b)=\sqrt{\frac{b-t}{t-a}} \chi_{[a, t]}(u) \frac{\sqrt{b-a}}{b-u}, \quad a<t<b,
$$

with the parameter $-\infty<a<b<\infty$. Then we shall show that the family $\mathscr{F}$ has an interesting relation with an element of $\operatorname{PGL}(2, \boldsymbol{R})$.

Theorem 3. (i) Let $\pi$ be a projective transformation from $[a, b]$ onto $[c, d]$ with the condition that $\pi(a)=c$ and $\pi(b)=d$. Then there exists an element $g$ of $P G L(2, \boldsymbol{R})$ such that the corresponding $g$ of $G_{0}$ (defined in Theorem 2) acts on $\mathcal{F}$ in the following way:

$$
\bar{g} f(\cdot ; t ; c, d)=f\left(\cdot ; \pi^{-1}(t) ; a, b\right), \quad c<t<d .
$$

(ii) For any two curves belonging to the family $\mathscr{F}$, there exist $\pi$ and $g$ which satisfy the relation (33).

(iii) Two stochastic processes 


$$
\left\langle x, f_{t}(\cdot ; t ; c, d)\right\rangle, \quad c<t<d,
$$

and

$$
\left\langle x, f\left(\cdot ; \pi^{-1}(t) ; a, b\right)\right\rangle, \quad c<t<d,
$$

have the some probability law.

Proof. We may consider $\pi$ the restriction of a linear transformation

$$
u \mapsto \frac{\alpha u+\beta}{\gamma u+\delta}
$$

to the interval $[a, b]$, which is also denoted by $\pi$. Let

$$
g=\left[\begin{array}{ll}
\alpha & \beta \\
\gamma & \delta
\end{array}\right]
$$

be the corresponding matrix in $G L(2, \mathbb{R})$. Since $\pi$ is projective it preserves the anharmonic ratio:

$$
(s, u ; a, b)=(\pi(s), \pi(u) ; c, d) \ldots, \cdots:
$$

Thus we have

$$
\frac{b-s}{s-a} \cdot \frac{u-a}{b-u}=\frac{d-\pi(s)}{\pi(s)-c} \cdot \frac{\pi(u)-c}{d-\pi(u)}, \quad a<s<b, a<u<b .
$$

Setting $t=\pi(s)$ and differentiating the expression (34) in $u$, we obtain

$$
\frac{b-\pi^{-1}(t)}{\pi^{-1}(t)-a} \cdot \frac{u-a}{b-u}=\frac{d-t}{t-c} \cdot \frac{\pi(u)-c}{d-\pi(u)}, \quad a<u<b, c<t<d
$$

and

$$
\frac{b-\pi^{-1}(t)}{\pi^{-1}(t)-a} \cdot \frac{b-a}{(b-u)^{2}}=\frac{d-t}{t-c} \cdot \frac{(d-c) \pi^{\prime}(u)}{(d-\pi(u))^{2}}, \quad a<u<b, c<t<d .
$$

Combining the formulas (34), (35) and (36), we have

$$
\begin{aligned}
\bar{g} f(u ; t ; c, d) & =\sqrt{\frac{d-t}{t-c}} \chi_{[c, t]}(\pi(u)) \frac{\sqrt{d-c}}{d-\pi(u)} \sqrt{\pi^{\prime}(u)} \\
& =\sqrt{\frac{b-\pi^{-1}(t)}{\pi^{-1}(t)-a}} \chi_{\left[a, \pi^{-i}(t)\right]}(u) \frac{\sqrt{b-a}}{b-u} \\
& =f\left(u ; \pi^{-1}(t) ; a, b\right) .
\end{aligned}
$$

The rest of the theorem is obvious.

Q.E. D.

Since 


$$
(f(\cdot ; t ; a, b), f(\cdot ; s ; a, b))=\sqrt{(s, t ; a, b)},
$$

the assertion (iii) of Theorem 3 means the principle of projective invariance of P. Lévy for the Brownian motion. The proof of Theorem 3 is an illustration of this principle in our set up.

\section{References}

[1] Гельқанд, И. М., Н. я. Вчленкци, Обобщенные Функдпи, Выпуск 4. Москва, 1961.

[2] Гельфанд, И. М., М. И. Граев, Н. Я. Ввленкпн. Обобщенные функпип. Выпуск 5, Москва, 1962.

[3] P. Lévy, Processus stochastiques et mouvement brownien, Gauthier-Villars, 1947. 
